\title{
FOREWORD
}

\section{Sir John Pendry FRS}

\author{
Guest Editors \\ John Inglesfield \\ School of Physics and \\ Astronomy, \\ Cardiff University, Cardiff, \\ CF24 3YB, UK
}

\section{Pedro Echenique}

Departamento de Física de Materiales y Centro Mixto UPV/CSIC, and Donostia

International Physics Center

(DIPC), Paseo de Manuel

Lardizabal 4, 20018

San Sebastián/Donostia,

Basque Country, Spain

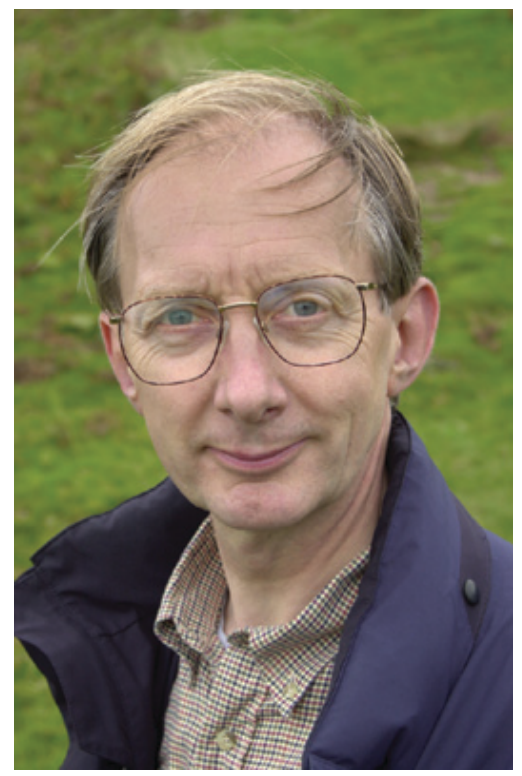

\section{John Inglesfield and Pedro Echenique write:}

John Pendry's 65th birthday is on 4 July 2008, and this issue of the Journal of Physics: Condensed Matter is dedicated to him, with articles by friends, colleagues, and former students. By any standards, John Pendry is a great scientist, who has made - and continues to make - an enormous contribution to physics; the wide range of his interests is reflected in the scope of these articles.

Not many scientists can establish a completely new and unexpected area of research, but this has been John's achievement in the last few years in the field of metamaterials, materials whose electromagnetic properties depend on their structure rather than the materials of which the structure is built. In this way, structures with effectively negative electrical permittivity and negative magnetic permeability can be constructed, demonstrating negative refraction; through metamaterials scientists now have access to properties not found in nature, and never previously explored experimentally. Never a week goes by without a potential new application of metamaterials, whether it is perfect lensing, or the cloak of invisibility. This has certainly led to tremendous visibility for John himself, with guest lectures all over the world, and radio and television appearances.

John Pendry's first paper was published exactly 40 years ago, 'Analytic properties of pseudopotentials' [1], and since then he has published 310 articles at the latest count. But this first paper already reflected something of the way John works. His PhD project, with Volker Heine at the Cavendish Laboratory, was to interpret the scattering of low energy electrons from surfaces, the technique of LEED which was to become the method of choice for determining surface structure. Although the energy of the electrons in LEED is relatively low-say 50 
$\mathrm{eV}$ - it is much higher than the energy of the conduction electrons, for which pseudopotentials had been devised, and John realised that he should explore the properties of pseudopotentials in depth. This had never been done before, and the result was a paper of typical originality. The story of how John explored truly original aspects of physics, right from the start of his research career, is given by Volker Heine (in his own inimitable style), at the end of this short biography.

Of course the result of John's PhD research was his development of the entire methodology for computing and interpreting LEED intensities, and their relationship to surface atomic structure. With experiments performed by Stig Andersson in Gothenburg, John's calculations led to the first ever surface structure determination, of $\mathrm{Na}$ adsorbed in a $\mathrm{c}(2 \times 2)$ structure on $\mathrm{Ni}(001)$ [2]. His 1974 book, 'Low Energy Electron Diffraction' [3], remains a classic, and not only for LEED theorists-there is plenty of other surface science here to get one's teeth into.

John extended the theory of LEED in the 1980s with the introduction of several new theoretical techniques and concepts. The Pendry R-factor [4] enabled surface structure determination to be largely automated, and quantified agreement between LEED theory and experiment. Tensor-LEED was developed by John, together with his $\mathrm{PhD}$ student Philip Rous [5], as an accurate approximation for calculating the LEED spectra of complex surface structures, enabling structures of hitherto impossible complexity to be determined. The methods of DLEED — both the experimental technique and its theoretical interpretation-were developed with Saldin, Van Hove and the Erlangen group of Heinz and Müller [6,7]; this is a technique for interpreting electron scattering from atoms randomly adsorbed on surfaces, hence determining their bonding to neighbouring atoms. LEED experiments and calculations continue to this day, of course, with John's contribution remaining fundamental. Articles in this issue by John's former PhD student Michel Van Hove, his one-time post-doc Dilano Saldin, and his long-standing collaborator Klaus Heinz, illustrate the power of LEED in determining surface structure. And everywhere in the surface science literature you will see the Pendry R-factor quoted, as a measure of the accuracy of the structure determination.

The contribution which theory and computation can make to the interpretation of electron spectroscopies has been an underlying theme in John's work over many years, and as in the case of LEED he has developed the computer programs to make the interpretation possible. After finishing his $\mathrm{PhD}$ in 1969, and during the period of a research fellowship at Downing College, Cambridge, John spent the year 1972-3 at Bell Laboratories in Murray Hill, New Jersey; it was there that he started collaborating with Patrick Lee on the interpretation of extended x-ray absorption fine structure (EXAFS) [8], oscillations seen above the absorption edge when x-rays excite core electrons of atoms in a molecular or solid state environment. This was the first quantitative theory of the effect, and led to the use of EXAFS in determining atomic structure in systems, such as glasses, where x-ray diffraction is less useful. Since then, EXAFS and its derivative techniques such as NEXAFS (which explores the structure immediately above the absorption edge, particularly sensitive to chemical bonding), and SEXAFS (EXAFS at surfaces) have become standard techniques in the armoury of structure determination methods at every synchrotron radiation laboratory.

And it was to the Daresbury Laboratory in Cheshire, with its synchrotron radiation facility, that John moved in 1975, from Cambridge, as Head of the Theory Group. This was an inspired appointment on the part of the then Science and Engineering Research Council, as it led to unrivalled theoretical support for the electron spectroscopists using the SRS facility. For John it was a move back to the North of England, the area from which he came and where he had been to school. In Daresbury he published his theory of angle-resolved photoemission [9], which remains the standard model in the field. These methods have enabled the band structure of electrons in solids and at surfaces to be determined to 
unprecedented accuracy, on the basis of photoemission experiments. But photoemission involves the occupied electronic states-what about the unoccupied states? These are just as important, certainly from the point of view of understanding the band structure and the many-body interaction with other electrons. In 1980 John proposed the technique of inverse photoemission, in which an electron emits light as it decays into the unoccupied states in the vicinity of the surface [10]. The viability of this technique was demonstrated soon afterwards, and it is now widely used for probing unoccupied electron states.

It was natural that John should explore the nature of electronic states at surfaces, and with Steve Gurman and later Pedro Echenique he developed criteria for the existence of the localised surface states, so important in determining surface properties and readily detectable using photoemission and inverse photoemission [11]. Their computational techniques were based on those used in the LEED and photoemission programs - the semi-infinite solid is split into atomic layers, the scattering properties of each layer are calculated, and then these are combined together to give the scattering of the solid with a surface. Such techniques are still immensely useful, and Simon Crampin has developed them further to calculate electronic states at surfaces with atomic islands, for example. This work began when John was still at Cambridge, but it was after his move to Daresbury that perhaps his most important work on surface states was produced - the demonstration of the possibility of resolving the image-potential-induced surface states on metal surfaces, with Echenique [12]. These states are caused by the image potential trapping an electron, which cannot penetrate into the bulk if its energy lies in a band-gap; the image states form a Rydberg series, and as their energy is just below the vacuum level, they are normally unoccupied. This makes inverse photoemission the ideal technique for observing them. Recent developments on two-photon photoemission, energy- and time-resolved, have produced a wealth of experimental information on these states. Since their prediction and observation, image states have become a playground for theoretical and experimental studies of many-electron effects at surfaces.

It is remarkable how John Pendry maintained his position as the leading theoretical surface physicist in the UK (and possibly the world) at the same time that he developed new areas of research. In 1981 John moved to Imperial College as Professor of Theoretical Solid State Physics and Head of the Condensed Matter Theory Group, and soon after began to study the behaviour of electrons in disordered systems, a field in which he collaborated with his colleague Angus MacKinnon. John applied group theoretical methods to study the transport of electrons in disordered media, and obtained for the first time a complete solution of the general scattering problem in one dimension [13], deriving advanced techniques for studying higher dimensions [14]. A key result was the prediction that in all dimensions, the channels for transport (distinct ways in which an electron can propagate through the system) are either open (that is, essentially transparent) or closed (opaque) in the limit of large systems [15]. These notions are relevant to such topical research as the conductivity of bio-molecules. In this novel approach to transport, John was helped by his wife Pat, who is a mathematician by training, with a thesis on group representation theory-John benefited not only from Pat's expertise, but also from her library on group theory, which was far better than that of most universities! (John and Pat wrote a joint paper shortly after they met in Cambridge, on scattering methods - a recurring theme in John's research [16].) What drove John to develop this new area of research, when the work on LEED and tensor-LEED was progressing so successfully? The answer is that he has always made a conscious effort to work on completely new topics, every ten years or so.

The question was what next? We remember John remarking about 20 years ago that the propagation of light in artificial periodic systems would be important in the future, and it turned out that the layer techniques he had developed for 
electronic structure could be adapted to electromagnetic waves (not so straightforward because of the vectorial nature of the electromagnetic field). As a result, and because of his appreciation of the importance of this field, John was working in photonics right from the start, and in 1994 he published his first papers on photonic band structures; his techniques enabled the interaction of light with metallic systems to be calculated, unlike the existing, poorly converging, plane wave methods. The titles of his early photonics papers show the originality of his work, and perhaps already indicate where it would eventually lead-'Refraction and geometry in Maxwell's equations' [17], 'Absolute three-dimensional photonic band gap in the infrared regime in woven structures' [18], 'Transmission resonances on metallic gratings with very narrow slits' [19], and many more. John went much further than most other researchers in the field, for example exploring the consequences of the near-field electromagnetic radiation, leading amongst other effects to friction between separated resistive plates, which could be associated with Van der Waal's forces [20]. And early on, he had invented the concept of metamaterials, devising a metallic mesostructure consisting of a three-dimensional array of wires with an extremely low plasmon frequency $[21,22]$.

Where it led to was the brilliant work on metamaterials which would show negative refraction [23,24], the concept of the perfect lens [25], ways of exploiting the near field for imaging purposes [26], methods of controlling electromagnetic fields and 'cloaks of invisibility' [27], and much more. His physical insight, as well as his mathematical virtuosity, was demonstrated early on in the story of metamaterials by the famous split-ring structure, with negative magnetic permeability as well as negative permittivity, and the way that John could solve Maxwell's equations for this system to give an effective $\epsilon$ and $\mu$ [23]. The split-ring resonator has been adopted in many laboratories as the starting point for their metamaterial designs. The work on the perfect lens sparked storms of enthusiasm and controversy in equal measure when it was first published in 2000, in the Physical Review Letter 'Negative refraction makes a perfect lens' [25]. Many eminent scientists demonstrated that the perfect lens, unlimited by conventional resolution criteria, was impossible, but of course it wasn't (we knew he was right!), and John's experimental colleagues have subsequently demonstrated the lensing effect. John's paper in 2000 has already been cited more than 1300 times, and was named letter-of-the-year: it can safely be said that the concept of the perfect lens has revolutionised nanoscale optics. His recent work with collaborators at Duke has shown that it is possible to design a 'cloak' that screens objects from electromagnetic fields; this resolved a long-standing mathematical challenge, questioning whether a region of space could be completely screened in this way [27]. Needless to say, this concept of a cloak of invisibility, which has been realised experimentally [28], has attracted great media interest, as well as numerous emails from schoolchildren.

John has always made a full contribution to wider scientific life, and during this latest, immensely productive period he has been Dean of the Royal College of Science (1993-1996), Head of Department at Imperial College (1998-2001), and subsequently Principal of the Faculty of Physical Sciences (2001- 2002). He has taken on the chairmanship of the Physics Sub-Panel of the 2008 UK Research Assessment Exercise, a huge task which he began in 2005. Elected a Fellow of the Royal Society in 1984, he has been Member of Council from 1992-1994, and Editor of the Royal Society Proceedings A from 1996-2002. For the Institute of Physics, since 2007 he has been Member of Council, Chairman of Institute of Physics Publishing and Vice-President for Publishing. He has received honours and awards, recognising his contributions, culminating in his knighthood for services to science in 2004, and the Royal Medal of the Royal Society in 2006.

John Pendry has worked with many research students, post-docs, and leading theoreticians and experimentalists, and has always been thoughtful and generous in his interactions with others. He is a very loyal person, loyal to friends, 
colleagues, his old college - it gave him great pleasure to be awarded an Honorary Fellowship at Downing College in 2005.

Beyond science there is that necessary hinterland, particularly his love and knowledge of music, for John is a fine pianist. We also think of his enjoyment of gardening, photography, the countryside, and natural history: hobbies shared with Pat. John relishes gadgets - the latest GPS or camera lens-perhaps his interest in photography kindled his interest in imaging? This is our friend John Pendry, whom we have had the privilege of knowing for 40 years - may he enjoy many more years of activity, research and the rest, in the years beyond his 65th birthday!

\section{Volker Heine ${ }^{1}$ writes:}

What I can contribute is a bit of history about John as a research student. After his Part II in Natural Sciences, he went on to do a one-year graduate diploma in advanced theoretical physics, called Part III of the Mathematics Tripos. I was away in Chicago on my first sabbatical leave, and Leo Falicov was spending a year in Cambridge holding the fort. So I asked him was this chap (JBP) really OK and should we take him, and Leo said 'yes'. So that was that.

The techniques of ultra high vacuum systems had been developed in the 1950s so that for the first time one could do experiments faster than dirt accumulated on a surface; and surface science took off. At that time before computing got into a useful stride, there were very few theoreticians around. As my professor in New Zealand expressed it to me when I said I wanted to do theory, he said there weren't any jobs in theoretical physics because it only took the Fermi's and Mott's of this world ten seconds to have an idea, and that was how it went. Indeed my first job in Cambridge was to 'demonstrate' (teach) in laboratory classes.

In the 1960s it was obvious that low energy electron diffraction (LEED) would clearly be extremely important for determining surface structures if one could only understand what it was telling one. X-ray and neutron diffraction were used to determine solid structures, and so why not electron diffraction for surfaces? Electrons in the $0-100 \mathrm{eV}$ range have a suitable wavelength. However they scatter extremely strongly from atomic potentials, and so the scattering pattern is much more complex than with $\mathrm{x}$-rays or neutrons from bulk, where each photon or neutron is only scattered once.

Well, we knew about solving the electronic structure for the conduction (valence) electrons in a solid, and so why not have a go in the LEED energy range. That was the project I suggested to John for his $\mathrm{PhD}$, and it worked. The only trouble was that the calculations took forever, because one had to solve the Schrödinger equation for the whole system of vacuum plus solid together: there didn't seem to be any short cut.

It was John who saw that there was a possible short cut. He is one of the few research students that I have had who did things independently that I could never have done myself. Although the forward scattering is indeed very strong, he noticed that the back scattering is much weaker, and this allowed him to develop a method of successive approximation. The material and the calculation are cut up into a series of atomic slices, and the electronic structure solved without approximation in each slice. Being two-dimensional, this was much more manageable than a full three-dimensional calculation. Then the slices could be put together by a sort of perturbation theory to calculate the scattering current as a function of energy along each of the reciprocal lattice 'rods' allowed by the surface periodicity.

John didn't just stop at having solved the problem in principle, he also developed a suite of computer codes to do the job in a routine way. He even published the code for all to use, and I believe that to the present day the calculations are still done effectively in the same way. Without the calculations, 
one cannot interpret the data in terms of a surface atomic structure. Effectively one is doing a computer experiment in parallel to the laboratory experiment, and one fiddles more or less systematically with the surface structure until one gets agreement between the simulations and the laboratory data.

One of the things that John noticed was that the process of photoemission (UPS) from a surface is rather similar, and can be calculated in an analogous way. This suddenly became very important because the new synchrotron at Daresbury was about to become operational, pumping out billions of photons for solid state research. Sam Edwards (now Sir Sam) was head of SRC (forerunner of EPSRC) at the time and recognised the problem of interpreting all the data that the machine would provide. And after UPS would come EXAFS, and after that XANES and all the rest of the alphabet soup. With that foresight, unusual at the time, John Pendry was appointed to head the theory group at Daresbury. Previously the theoreticians had revolved around the nuclear physics accelerator, and John had to turn it completely around and refocus it, which he did, ably supported and abetted by Phil Burke as head of the Theory and Computational Science Division.

1 (Cavendish Laboratory, J J Thomson Avenue, Cambridge CB3 OHE, UK)

\section{Selected Bibliography}

[1] Pendry J B 1968 J. Phys. C: Solid State Phys. 1 1065-74

[2] Andersson S and Pendry J B 1972 J. Phys. C: Solid State Phys. 5 L41-5

[3] Pendry J B 1974 Low Energy Electron Diffraction (London: Academic Press)

[4] Pendry J B 1980 J. Phys. C: Solid State Phys. 13 937-44

[5] Rous P J and Pendry 1989 Surf. Sci. 219 355-72

[6] Saldin D K, Pendry J B, Van Hove M A and Somorjai G A 1985 Phys. Rev. B 31 1216-8

[7] Heinz K, Saldin D K and Pendry J B 1985 Phys. Rev. Lett. 55 2312-5

[8] Lee P A and Pendry J B 1975 Phys. Rev. B 11 2795-811

[9] Pendry J B 1976 Surf. Sci. 57 679-705

[10] Pendry J B 1980 Phys. Rev. Lett. 45 1356-8

[11] Pendry J B and Gurman S J 1975 Surf. Sci. 49 87-105

[12] Echenique P M and Pendry J B 1978 J. Phys. C: Solid State Phys. 11 2065-75

[13] Slevin K M and Pendry J B 1988 J. Phys. C: Solid State Phys. 21 141-9

[14] Pendry J B and Castaño E 1988 J. Phys. C: Solid State Phys. 21 4333-55

[15] Pendry J B, MacKinnon A and Prêtre A B 1990 Physica A 168 400-7

[16] Pendry J B and Gard P 1975 J. Phys. C: Solid State Phys. 8 2048-58

[17] Ward A J and Pendry J B 1996 J. Mod. Opt. 43 773-93

[18] Tsai Y-C, Pendry J B and Shung K W-K 1999 Phys. Rev. B 59 15261-6

[19] Porto J A, García-Vidal F J and Pendry J B 1999 Phys. Rev. Lett. 83 2845-8

[20] Pendry J B 1997 J. Phys.: Condens. Matter 9 10301-20

[21] Pendry J B, Holden A J, Stewart W J and Youngs I 1996 Phys. Rev. Lett. 76 4773-6

[22] Pendry J B, Holden A J, Robbins D J and Stewart W J 1998 J. Phys.: Condens. Matter 10 4785-809

[23] Pendry J B, Holden A J, Robbins D J and Stewart W J 1999 IEEE Trans. Microw. Theory Tech. $472075-84$

[24] Smith D R, Pendry J B and Wiltshire M C K 2004 Science 305 788-92

[25] Pendry J B 2000 Phys. Rev. Lett. 85 3966-9

[26] Wiltshire M C K, Hajnal J V, Pendry J B, Edwards D J and Stevens C J 2003 Opt. Express 11 $709-15$

[27] Pendry J B, Schurig D and Smith D R 2006 Science 312 1780-2

[28] Schurig D, Mock D D, Justice B J, Cummer S A, Pendry J B, Starr A F and Smith D R 2006 Science 314 977-80 